\title{
Vascularized Lymph Node Transfer for the Treatment of Posttraumatic Lower Extremity Lymphedema: A Preliminary Report
}

\author{
Pedro Ciudad ${ }^{1,2}$ Maria T. Huayllani ${ }^{3}$ Antonio J. Forte ${ }^{3(0)}$ Daniel Boczar $^{3}$ Francisco R. Avila ${ }^{3}$ \\ Joseph M. Escandón ${ }^{4}$ Oscar J. Manrique ${ }^{4}$ Hung-Chi Chen ${ }^{2}$
}

\footnotetext{
${ }^{1}$ Department of Plastic, Reconstructive and Burn Surgery, Arzobispo Loayza National Hospital, Lima, Peru

${ }^{2}$ Department of Plastic and Reconstructive Surgery, China Medical University Hospital, Taichung, Taiwan

${ }^{3}$ Division of Plastic Surgery, Mayo Clinic, Jacksonville, Florida, United

States

${ }^{4}$ Division of Plastic and Reconstructive Surgery, Strong Memorial Hospital, University of Rochester Medical Center, Rochester, New York, United States
}

Indian J Plast Surg 2022;55:97-101.

\author{
Address for correspondence Hung-Chi Chen, Department of Plastic \\ and Reconstructive Surgery, China Medical University Hospital, \\ Taichung, Taiwan (e-mail: d19722@mail.cmuh.org.tw).
}

\begin{abstract}
Keywords

- lymphedema

- VLNT

- lymph nodes/ transplantation

Background Posttraumatic lymphedema develops more frequently than expected and reports on its management are scarce in the literature. We aimed to report the clinical outcomes of a case series of posttraumatic lymphedema patients treated with different vascularized lymph node transfers (VLNTs).

Patients and Methods Five patients with secondary posttraumatic lower limb lymphedema treated with VLNT were included in this report. The groin-VLNT $(n=1)$, supraclavicular-VLNT $(n=2)$, and gastroepiploic-VLNT $(n=2)$ were implemented. The average flap area was $69.8 \mathrm{~cm}^{2}$. Patients underwent postoperative complex decompressive therapy for an average of 10.0 months.

Results The average mean circumference reduction rate was $24.4 \%$ (range, 10.2$37.6 \%$ ). Postsurgical reduction in the number of infection episodes per year was observed in all patients. The mean follow-up was 34.2 months.

Conclusions VLNT is a promising surgical treatment for posttraumatic lymphedema patients. In our report, VLNT has demonstrated to reduce the volume and number of infections per year in posttraumatic lymphedema.
\end{abstract}

\section{Introduction}

Wound healing consists of an early proinflammatory response in early stages, followed by a proliferative and remodeling phase. However, in some instances after extensive trauma in which the integrity of soft tissue and bone is severely compromised, insufficient circulation, chronic infections, and a long-lasting inflammatory state can halt the normal progression of wound healing. ${ }^{1,2}$ In this setting, the obstruction of lymphatic channels, a continuous inflammatory milieu, and abnormal scarring can cause persistent lymphedema, which has been recognized in 20 to $25 \%$ of published online

February 28, 2022
DOI https://doi.org/ 10.1055/s-0041-1740520. ISSN $0970-0358$.

\footnotetext{
(c) 2022. Association of Plastic Surgeons of India. All rights reserved. This is an open access article published by Thieme under the terms of the Creative Commons Attribution-NonDerivative-NonCommercial-License, permitting copying and reproduction so long as the original work is given appropriate credit. Contents may not be used for commercial purposes, or adapted, remixed, transformed or built upon. (https://creativecommons.org/ licenses/by-nc-nd/4.0/)

Thieme Medical and Scientific Publishers Pvt. Ltd., A-12, 2nd Floor, Sector 2, Noida-201301 UP, India
} 
cases following traumatic bone fractures. ${ }^{1}$ Due to irreversible alterations in the lymphatic system, obtaining optimal results when restoring the physiologic lymphatic flow may be problematic. Therefore, from an anatomophysiological standpoint, a comprehensive reconstruction of the lymphatic system may be necessary beyond solely restoring the flow of lymphatic fluid.

Vascularized lymph node transfer (VLNT) is a microsurgical technique currently used to treat lymphedema in which lymph nodes are transferred from a healthy donor site to an affected lymphedematous limb. ${ }^{3,4}$ VLNT has demonstrated positive outcomes, specifically when lymphatics are not available for a lymphaticovenous anastomosis (LVA). ${ }^{5}$ Additionally, it has shown to promote lymphangiogenesis, reduce fibrosis, and decrease inflammatory markers. ${ }^{3,4,6}$ Therefore, VLNT may be considered as a potential microsurgical procedure for patients who develop posttraumatic lymphedema. Herein, we report the clinical outcomes of five patients with posttraumatic lymphedema of the lower extremities who underwent VLNT from diverse donor sites.

\section{Patients and Methods}

From July 2010 to July 2016, patients with posttraumatic lower limb lymphedema were treated with VLNT at the China Medical University Hospital. Diagnosis was based on clinical evaluation, and lymphedema was classified according to the International Society of Lymphology (ISL). Lymphoscintigraphy of the lower extremities with evidence of dermal backflow and lymphatic obstruction confirmed the diagnosis. All patients received initial complex decompressive therapy (CDT). Five patients who did not show response to CDT for at least 6 months and who had at least 2 years of follow-up were included in our report. Demographic data and clinical pre- and postoperative photographs were documented ( - Fig. $\mathbf{1}$ ). The average age was 28.2 years (range, 27 31 ). One patient needed skin coverage, and other two needed external fixation of the lower limb after trauma. All patients developed posttraumatic lower limb lymphedema after a car accident. Three patients developed stage IIB lymphedema, while two developed lymphedema stage III ( - Table 1). Circumferences of the lymphedematous extremity and the unaffected counterpart before and at 3, 6, 12 and 24 months after VLNT were recorded. The anatomic landmarks used to measure the circumference were the following: $10 \mathrm{~cm}$ below the knee, $10 \mathrm{~cm}$ above the ankle, and at the midfoot. To calculate the circumference reduction rate, we used the mean of the measured circumferences. ${ }^{7,8}$ We defined the percentage of improvement as "reduction rate" comparing the affected limb (AL) to the healthy limb (HL) as follows: circumference reduction rate $(\%)=(1-$ [postoperative $\mathrm{AL}$ $\mathrm{HL}] \times 100$ [pre - operative AL $-\mathrm{HL}]$ ).

\section{Surgical Technique}

VLNT was performed as previously reported. ${ }^{7}$ Antibiotics were administered 1 hour before surgery and continued postoperatively for 3 days. The dorsalis pedis or the medial plantar and their concomitant veins were used as recipient vessels. The donor site for VLNT were the groin $(n=1)$, supraclavicular $(n=2)$, and gastroepiploic regions $(n=2)$.

When groin- or supraclavicular-VLNT were employed, the skin paddle and a split-thickness skin graft (STSG) were utilized to cover the defect. A skin island was designed approximately $2 \mathrm{~cm}$ below the inguinal ligament and lateral to the femoral artery for the groin-VLNT. The flap could be based on the superficial circumflex iliac artery (SCIA), superficial epigastric artery, medial branch of femoral artery, or the reverse flow of the SCIA. ${ }^{9}$ When the right supraclavicular lymph node flap (LNF) was harvested, an incision was made $1.5 \mathrm{~cm}$ above the clavicle and a freestyle LNF was elevated based on the transverse cervical artery (TCA). ${ }^{7}$ The concomitant veins were identified and included within the flap, while the external jugular vein was also included to improve the venous drainage. ${ }^{10}$

The right gastroepiploic artery and vein were used for GEVLNT (-Fig. 2). The flap was harvested through an upper midline laparotomy incision. The omentum was carefully dissected out of the transverse colon, avoiding injuries to the mesocolon. The left gastroepiploic vessels were then ligated and divided. Dissection of the short segmental gastric
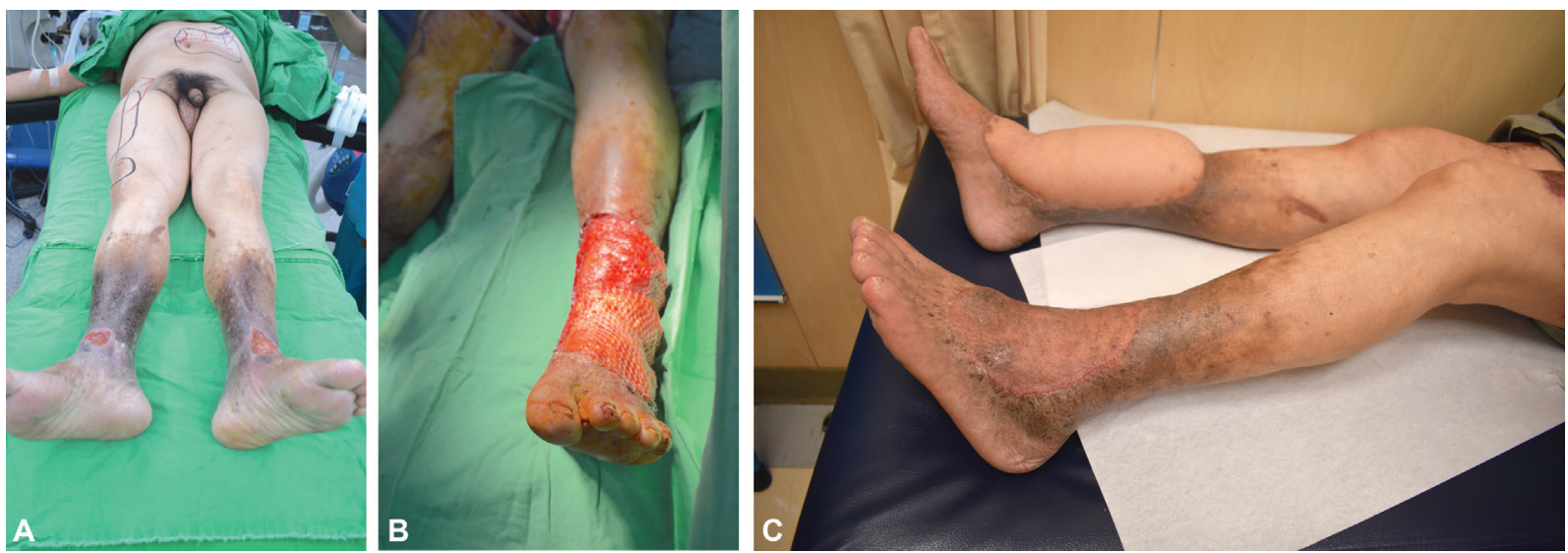

Fig. 1 Posttraumatic lymphedema managed with a gastroepiploic-vascularized lymph node transfer (GE-VLNT). (A) 50 year-old male undergoing simultaneous ALT and GE-VLNT; (B) Flap inset of the left lower limb; (C) Postoperative picture of the lower limbs at 1 year of follow-up. ALT, anterolateral thigh; GE-VLNT, gastroepiploic-vascularized lymph node transfer. 


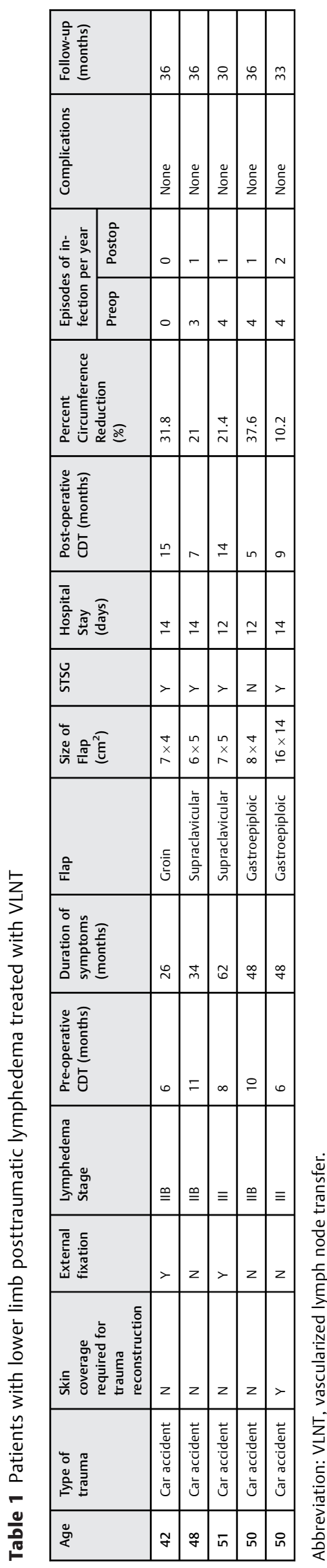

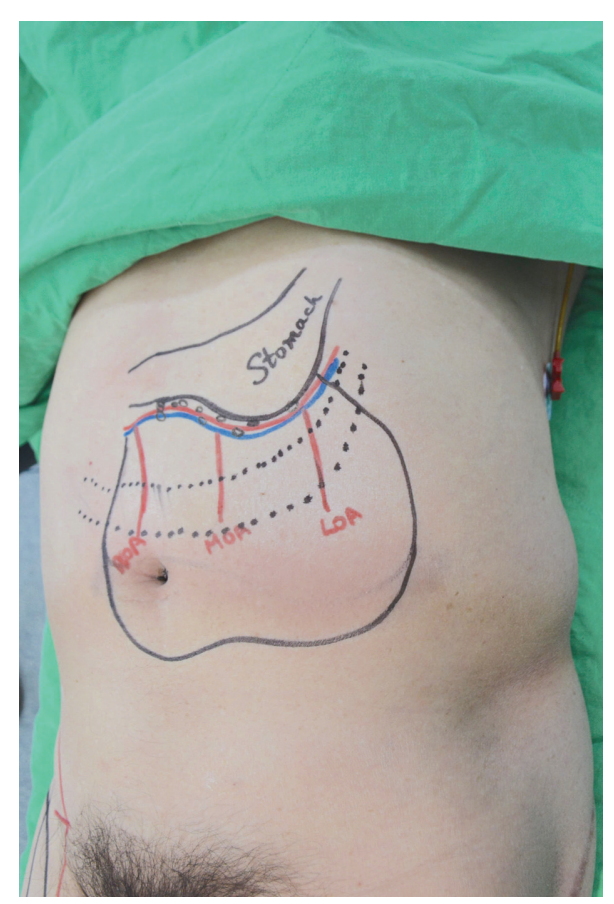

Fig. 2 Gastroepiploic-vascularized lymph node transfer (GE-VLNT) preoperative markings.

branches allowed further release of the flap from the stomach and permitted complete visualization of the gastroepiploic vessels ( - Fig. 3). Dissection continued to the level of the right epiploic vessels. The lymph nodes within the flap cannot always be visualized, but they often can be palpated. Indocyanine green lymphatic imaging may be performed to confirm the vascularity of the lymph nodes included within the flap. ${ }^{3,7,9}$ In one patient, the gastroepiploic-VLNT required coverage with a STSG, while rotational skin flaps were sufficient to cover the other patient's flap.

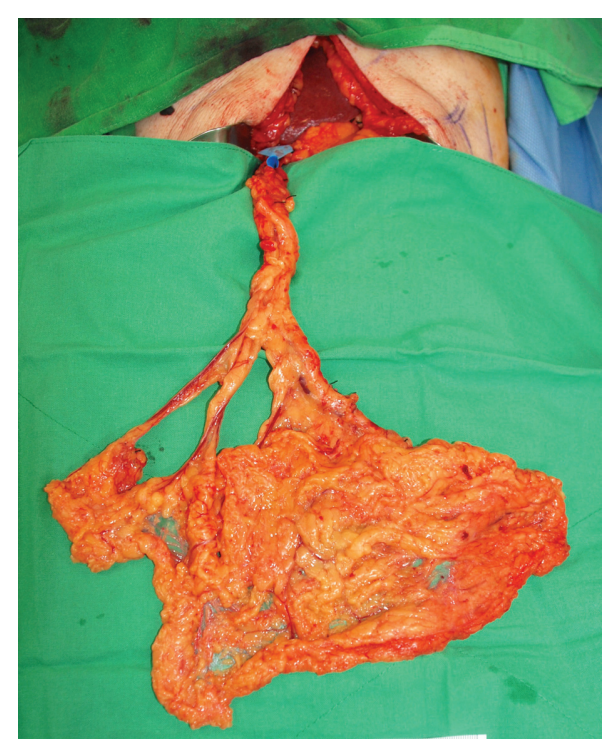

Fig. 3 Intraoperative picture of the right gastroepiploic-vascularized lymph node transfer (GE-VLNT). 


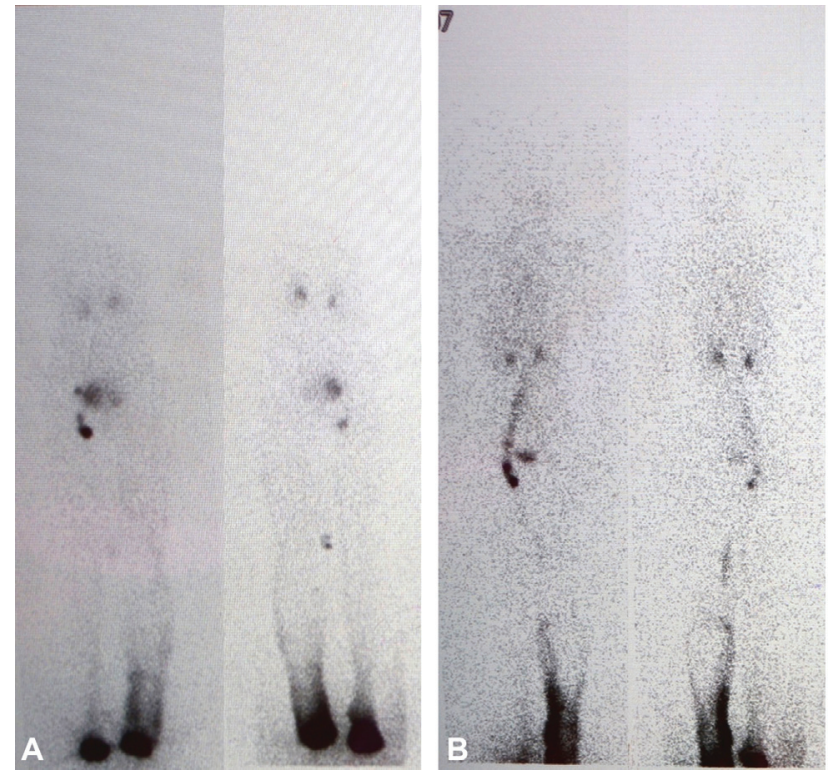

Fig. 4 Postoperative improvement in the lymphatic drainage evidenced using lymphoscintigraphy. (A) preoperative; (B) postoperative.

\section{Postoperative Care}

Patients were encouraged to keep the leg elevated, and deep venous thrombosis prophylaxis was administered postoperatively. At 4 weeks postsurgery, once the VLNT healed and viability was confirmed, all patients continued with CDT and rehabilitation. All patients were evaluated through lymphoscintigraphy at 1 and 2 years postoperative (-Fig. 4).

\section{Results}

The average duration of symptoms and preoperative CDT was 43.6 months (range, 26-62 months) and 8.2 months (range, 6-11 months), respectively. The average area of flaps was $69.8 \mathrm{~cm}^{2}$ (range, $28-224 \mathrm{~cm}^{2}$ ). Only one patient did not need a STSG to cover the VLNT. The average hospitalization duration was 13.2 days (range, 12-14 days). No postoperative complications were reported. Postoperative CDT was performed for 10.0 months (range, 5-15 months). The average follow-up was 34.2 months (range, 30-36). The mean postoperative circumference reduction was $24.4 \%$ (range, $10.2-$ $37.6 \%$ ). All patients were able to ambulate postoperatively and experienced a reduction in the number of infection episodes per year, if reported preoperatively. Besides undergoing a GE-VLNT for a posttraumatic lymphedematous limb, one patient underwent soft-tissue coverage of a nonlymphedematous limb with an anterolateral thigh flap, reporting symptomatic improvement of venous insufficiency (- Fig.1).

\section{Discussion}

As described by Szczesny et al, alterations in the lymphatic drainage have a main role in the incidence of posttraumatic edema, as even with preservation of the superficial lymphatic system, edema can occur in virtually all cases due to decreased lymphatic flow and increased production of lymphatic fluid, secondary to inflammatory mechanisms and irritation by heme-containing compounds. ${ }^{1,2,11}$ Alterations in the venous outflow were initially thought to be the main pathophysiologic mechanism; however, venous thromboembolism was only recognized in $24 \%$ of the subjects developing posttraumatic edema. ${ }^{1,2}$ In these cases, lymphoscintigraphy gains an important value during the evaluation of posttraumatic edema, in which an increase in lymphatic outflow and contrast in lymph nodes, and extravasation of contrast into the skin can confirm the diagnosis of lymphedema. ${ }^{12}$

CDT is the first line of treatment for lymphedema. When CDT does not result in successful outcomes, physiologic surgical options (LVA or VLNT) can be considered. LVA has been previously applied in patients with posttraumatic lymphedema. Olszewski et al reported circumference reduction rates of $30 \%$ to $40 \%$ in 770 lower limb postinflammatory/ posttraumatic lymphedema patients treated with LVA. ${ }^{13}$ However, in cases in which nonfunctional lymphatic ducts may be present, such as in trauma cases, VLNT may increase the remission rate. ${ }^{13}$ In fact, VLNT has shown positive outcomes in cases in which LVA cannot be performed, with satisfactory circumference reduction rates. ${ }^{14,15}$ Ciudad et al described the case of a 33-year-old man with posttraumatic lower limb lymphedema treated with a GE-VLNT. ${ }^{16}$ The patient had a circumference reduction rate of $36.5 \%, 32 \%, 21.4 \%$, and $15.2 \%$ above the knee, below the knee, above the ankle, and at the foot, respectively, at 15 months of follow-up. ${ }^{16}$ In our report, we found a lower mean circumference reduction of $24.4 \%$ at a mean of 34 months of follow-up. Moreover, we identified that three out of five patients with greater circumference reduction rates also had longer periods of preoperative and postoperative CDT. Indicating CDT is a crucial factor that may have expediated an enhanced recovery by means of a synergic effect with the physiologic surgical procedures in the setting of trauma.

Localization of the lymphedema plays a fundamental role in surgical outcomes. ${ }^{5}$ Becker et al presented two cases of posttraumatic lymphedema in the upper extremity treated with VLNT. They reported complete lymphedema remission after surgery. ${ }^{17}$ Conversely, we could not observe complete lymphedema remission at 2 years of follow-up. These findings were attributed to the impact of gravitational forces in the lower limb, which makes it harder to accomplish a total remission of lymphedema compared with upper extremity lymphedema. Disease staging was another factor that influenced our results, since we included patients with ISL stage IIB or III. Therefore, as identified in previous reports, lower circumference reduction rates were expected in comparison to previous reports. ${ }^{5}$

In our experience, the GE-VLNT is preferred for most cases due to the inexistent risk of iatrogenic lymphedema and because of the abundant number of nodes, which allows for a double inset by separating the flap. However, we believe the use of supraclavicular and groin LNFs with a skin paddle might be more beneficial to cover any traumatic defect. It is also important to note that when performing a VLNT, a skin paddle flap can favor the reestablishment of the lymphatic drainage through an additional angiogenic 
network of the afferent lymphatic vessels, from the dermis of the flap's skin paddle, and the recipient lymphatic ducts. Moreover, this can also favor the development of new lymphatic vessels through the process of lymphangiogenesis on the long-term. Furthermore, we observed that the number of skin infections per year decreased after surgery for all patients who had infections preoperatively, in accordance with previous reports in which VLNT decreased the frequency of infections in patients with posttraumatic lymphedema. ${ }^{18}$

To our knowledge, this is the first report that describes the use of multiple donor sites for VLNT to treat posttraumatic lymphedema. Our findings suggest VLNT could be potentially considered at approximately 6 months after unsuccessful CDT, as it will reduce volume and number of infections in posttraumatic lymphedema. An early diagnosis of posttraumatic lymphedema can prevent worsening of the disease and improve surgical outcomes and prognosis. Further studies need to evaluate and compare surgical outcomes with other causes of secondary lymphedema treated with VLNT to evaluate its efficacy. Despite the positive outcomes shown by VLNT, lymphedema can still recur, especially in more advanced stages of disease as previously reported. ${ }^{19}$ Certainly, donor site selection for VLNT based on the availability, recipient location, morbidity, preference of the patient, and surgeon's comfort and experience may also improve outcomes for this complex and underreported entity.

\section{Conclusions}

VLNT is a feasible option for posttraumatic lymphedema management when CDT does not improve lymphatic drainage. From a pathophysiologic viewpoint, VLNT has shown to be beneficial in reducing limb volume and number of infections per year in patients with posttraumatic lymphedema due to its pumping mechanism, lymphangiogenic capacity, and antioxidative potential.

\section{Financial Disclosure and Products}

None.

\section{Funding Source}

This report was supported in part by the Mayo Clinic Center for Individualized Medicine and the Plastic Surgery Foundation. (C2020 Mayo Foundation for Medical Education and Research

\section{Conflict of Interest}

None declared.

\section{References}

1 Szczesny G, Olszewski WL. The pathomechanism of posttraumatic edema of lower limbs: I. The effect of extravasated blood, bone marrow cells, and bacterial colonization on tissues, lymphatics, and lymph nodes. J Trauma 2002;52(02):315-322
2 Szczesny G, Veihelmann A, Nolte D, Messmer K. Changes in the local blood and lymph microcirculation in response to direct mechanical trauma applied to leg: in vivo study in an animal model. J Trauma 2001;51(03):508-517

3 Ciudad P, Maruccia M, Socas J, et al. The laparoscopic right gastroepiploic lymph node flap transfer for upper and lower limb lymphedema: technique and outcomes. Microsurgery 2017;37(03):197-205

4 Ciudad P, Sabbagh MD, Agko M, et al. Surgical management of lower extremity lymphedema: A comprehensive review. Indian J Plast Surg 2019;52(01):81-92

5 Ciudad P, Manrique OJ, Bustos SS, et al. Comparisons in long-term clinical outcomes among patients with upper or lower extremity lymphedema treated with diverse vascularized lymph node transfer. Microsurgery 2020;40(02):130-136

6 Viitanen TP, Visuri MT, Sulo E, Saarikko AM, Hartiala P. Antiinflammatory effects of flap and lymph node transfer. J Surg Res 2015;199(02):718-725

7 Ciudad P, Agko M, Perez Coca JJ, et al. Comparison of long-term clinical outcomes among different vascularized lymph node transfers: 6-year experience of a single center's approach to the treatment of lymphedema. J Surg Oncol 2017;116(06):671-682

8 Ciudad P, Forte AJ, Huayllani MT, et al. Impact of body mass index on long-term surgical outcomes of vascularized lymph node transfer in lymphedema patients. Gland Surg 2020;9(02):603-613

9 Ciudad P, Manrique OJ, Date S, et al. A head-to-head comparison among donor site morbidity after vascularized lymph node transfer: pearls and pitfalls of a 6-year single center experience. J Surg Oncol 2017;115(01):37-42

10 Chilgar RM, Khade S, Chen HC, et al. Surgical treatment of advanced lymphatic filariasis of lower extremity combining vascularized lymph node transfer and excisional procedures. Lymphat Res Biol 2019;17(06):637-646

11 Wandolo G, Elias RM, Ranadive NS, Johnston MG. Heme-containing proteins suppress lymphatic pumping. J Vasc Res 1992;29 (03):248-255

12 Pun WK, Chow SP, Fang D, Cheng CL, Leong JCY, Ng C. Posttraumatic oedema of the foot after tibial fracture. Injury 1989;20 (04):232-235

13 Olszewski WL. Lymphovenous microsurgical shunts in treatment of lymphedema of lower limbs: a 45-year experience of one surgeon/one center. Eur J Vasc Endovasc Surg 2013;45(03): 282-290

14 Pappalardo M, Patel K, Cheng MH. Vascularized lymph node transfer for treatment of extremity lymphedema: An overview of current controversies regarding donor sites, recipient sites and outcomes. J Surg Oncol 2018;117(07):1420-1431

15 Engel H, Lin CY, Huang JJ, Cheng MH. Outcomes of lymphedema microsurgery for breast cancer-related lymphedema with or without microvascular breast reconstruction. Ann Surg 2018; 268(06):1076-1083

16 Ciudad P, Manrique OJ, Agko M, et al. Ileocecal vascularized lymph node transfer for the treatment of extremity lymphedema: a case report. Microsurgery 2019;39(01):81-84. Doi: 10.1002/micr.30186

17 Becker C, Arrivé L, Mangiameli G, Pricopi C, Randrianambinina F, Le Pimpec-Barthes F. Post-traumatic massive hand lymphedema fully cured by vascularized lymph node flap transfer. SICOT J 2018;4:53. Doi: 10.1051/sicotj/2018049

18 Mihara M, Hara H, Kikuchi K. Lymph node transfer for refractory infectious sites caused by trauma. SAGE Open Med Case Rep 2017; 5:X17711631

19 Ciudad P, Date S, Manrique OJ, et al. Recurrent advanced lower extremity lymphedema following initial successful vascularized lymph node transfer: a clinical and histopathological analysis. Arch Plast Surg 2017;44(01):87-89 